\title{
DETERMINANT EXPRESSIONS FOR ABELIAN FUNCTIONS IN GENUS TWO
}

\author{
YOSHIHIRO ÔNISHI \\ Faculty of Humanities and Social Sciences, Iwate University, Morioka, 020-8550, Japan \\ e-mail:onishi@iwate-u.ac.jp
}

(Received 30 October, 2000; accepted 5 November, 2001)

\begin{abstract}
In this paper we generalize the formula of Frobenius-Stickelberger (see $(0.1)$ below) and the formula of Kiepert (see (0.2) below) to the genus-two case.

2000 Mathematics Subject Classification. 11G30, 11G10, 14H45.

Introduction. There is a classical formula

$$
\begin{aligned}
& (-1)^{n(n-1) / 2} 1 ! 2 ! \cdots n ! \frac{\sigma\left(u_{0}+u_{1}+\cdots+u_{n}\right) \prod_{i<j} \sigma\left(u_{i}-u_{j}\right)}{\sigma\left(u_{0}\right)^{n+1} \sigma\left(u_{1}\right)^{n+1} \cdots \sigma\left(u_{n}\right)^{n+1}} \\
& =\left|\begin{array}{cccccc}
1 & \wp\left(u_{0}\right) & \wp^{\prime}\left(u_{0}\right) & \wp^{\prime \prime}\left(u_{0}\right) & \cdots & \wp^{(n-1)}\left(u_{0}\right) \\
1 & \wp\left(u_{1}\right) & \wp^{\prime}\left(u_{1}\right) & \wp^{\prime \prime}\left(u_{1}\right) & \cdots & \wp^{(n-1)}\left(u_{1}\right) \\
\vdots & \vdots & \vdots & \vdots & \ddots & \vdots \\
1 & \wp\left(u_{n}\right) & \wp^{\prime}\left(u_{n}\right) & \wp^{\prime \prime}\left(u_{n}\right) & \cdots & \wp^{(n-1)}\left(u_{n}\right)
\end{array}\right|
\end{aligned}
$$

where $\sigma(u)$ and $\wp(u)$ are the usual elliptic functions. As far as the author knows the earliest work in which this formula appeared is a paper of Frobenius and Stickelberger [10]. Before publication of their paper, Kiepert [14] gave the formula

$$
(-1)^{n(n-1) / 2}(1 ! 2 ! \cdots(n-1) !)^{2} \frac{\sigma(n u)}{\sigma(u)^{n^{2}}}=\left|\begin{array}{cccc}
\wp^{\prime}(u) & \wp^{\prime \prime}(u) & \cdots & \wp^{(n-1)}(u) \\
\wp^{\prime \prime}(u) & \wp^{\prime \prime \prime}(u) & \cdots & \wp^{(n)}(u) \\
\vdots & \vdots & \ddots & \vdots \\
\wp^{(n-1)}(u) & \wp^{(n)}(u) & \cdots & \wp^{(2 n-3)}(u)
\end{array}\right|
$$

which also can be obtained by a limiting process from $(0.1)$. These formulae are included in the book [21, p. 458 and p. 460] as exercises and are also treated in [9, I, p. 183 and p. 186]. The function $\sigma(n u) / \sigma(u)^{n^{2}}$ in $(0.2)$ has been denoted by $\psi_{n}(u)$ ever since the book of Weber [20].

There is also a detailed analytic theory of Abelian functions for the Jacobian variety of any hyperelliptic curve. In the theory there is a nice generalization by H.F. Baker and others of the elliptic sigma function. If the genus of the curve, say $C$, is $g$, this function is also denoted by $\sigma(u)=\sigma\left(u^{(1)}, \cdots, u^{(g)}\right)$ (see (1.1) below). Although the function

$$
\frac{\sigma(n u)}{\sigma(u)^{n^{2}}}
$$


is a function on the Jacobian variety and is a natural generalization of $\psi_{n}(u)$ for the given genus $g$ curve, it has poles along a theta divisor $\Theta$ which is the $(g-1)$-fold sum of the curve $C$ embedded with a Weierstrass point as base point, so does not naturally restrict to a function on the curve itself.

Now we restrict ourselves to consider only functions associated to a hyperelliptic curve of genus $g=2$. Then we consider the modified function

$$
\frac{\sigma(n u)}{\sigma_{2}(u)^{n^{2}}},
$$

where $\sigma_{2}(u)=\left(\partial \sigma / \partial u^{(2)}\right)(u)$. Although this function is not a function on the Jacobian variety of the curve, via restriction to $C$ it can be regarded as a function on the curve itself, and is a good candidate to be called the hyperelliptic psi function. So we denote it by $\psi_{n}(u)$. The zeroes of $\psi_{n}(u)$ on the curve are just the points whose multiplication by $n$ are on $\Theta$. If $g=2$ the theta divisor $\Theta$ coincides exactly with $C$.

The aim of this paper is to give a natural generalization of the expressions $(0.1)$ and (0.2) for any hyperelliptic curve of genus two. See Theorem 2.3 and Theorem 3.3 below.

The function $\psi_{n}(u)$ has been investigated in several contexts as follows. First, it had an important role in the new theory of complex multiplication due to D. Grant ([12] and [19]). Secondly, a recursion relation for any hyperelliptic psi function was given by D.G. Cantor in [7], and such a relation is used to compute the torsion points on the corresponding curve. He also gave a determinant expression for $\psi_{n}(u)$, which is different from our expression and should be regarded as a generalization of the formula of Brioschi $[\mathbf{6}$, p. 770, 1.3] to all hyperelliptic curves.

After this paper had been already prepared, our formula of Kiepert type was generalized to all hyperelliptic curves by S. Matsutani and the author in [15] by using the formula of Cantor.

The formula (0.1) also has a classical generalization to all non-singular algebraic curves in terms of the Klein prime form, which is the formula (44) in [8, p. 33], and a generalization to all hyperelliptic curves of genus two in terms of Gunning's prime form, which is the formula (2) of [13]. The referee of this paper kindly informed the author about these two kinds of generalizations.

The author would like to acknowledge that the proof of the main theorem (Theorem 2.3) was simplified by the referee.

The first things which inspired the author in this work are the very important and strange formulae which appeared in the famous papers [16] and [17]. These papers solved the problem of the determination of the argument of cubic and quartic Gauss sums, respectively, by using the formulae given by Propositions 7.3 and 7.4 in [16, p. 181] and by (6.37) and (6.38) in [17], which resemble quite closely (0.2) above. Matthews mentions in [16, p. 179] that such a line of attack was suggested by observations of S.J. Patterson. The author hopes that our new formulae will be used to establish a new theory of complex multiplication.

The author was led to believe in the existence of our determinant expressions through continual communication with Matsutani. The author found another generalization of the formula of Frobenius and Stickelberger to the three variables case (i.e. $n=2$ ) for genus-two curves in [5, pp. 96-97] after having already obtained these results. Quite recently all our formulae were generalized to any hyperelliptic curves by the author himself in [19]. Moreover the connection of Theorem 3.3 below and the determinant expression of Cantor mentioned above was completely clarified by S. Matsutani (see Appendix of [19]). 
0. Convention. We denote, as usual, by $\mathbf{Z}$ and $\mathbf{C}$ the ring of rational integers and the field of complex numbers, respectively. In an expression of the Laurent expansion of a function, the symbol $\left(d^{\circ}\left(z_{1}, z_{2}, \cdots, z_{m}\right) \geq n\right)$ stands for the terms of total degree at least $n$ with respect to the given variables $z_{1}, z_{2}, \cdots, z_{m}$.

In cross references, we indicate a formula as (1.2), and each of Lemmas, Propositions, Theorems and Remarks also as 1.2.

1. The sigma function in genus two. In this Section we summarize the fundamental facts used in Sections 2 and 3. Detailed treatment of these facts are given in [1], [2] and [3] (see also Section 1 of [19]).

Let

$$
f(x)=\lambda_{0}+\lambda_{1} x+\lambda_{2} x^{2}+\lambda_{3} x^{3}+\lambda_{4} x^{4}+\lambda_{5} x^{5}
$$

be an polynomial of $x$ over $\mathbf{C}$ such that all its zeros are different from each other. Let $C$ be a smooth projective model of the curve of genus 2 defined by $y^{2}=f(x)$. We denote by $\infty$ the unique point at infinity. In this paper we suppose $\lambda_{5}=1$. The set of forms

$$
\omega^{(1)}=\frac{d x}{2 y}, \omega^{(2)}=\frac{x d x}{2 y}
$$

is a basis of the space of differential forms of first kind. Let

$$
\eta^{(1)}=\frac{\left(\lambda_{3} x+2 \lambda_{4} x^{2}+3 \lambda_{5} x^{3}\right) d x}{2 y}, \quad \eta^{(2)}=\frac{x^{2} d x}{2 y} .
$$

Then $\eta^{(1)}$ and $\eta^{(2)}$ are differential forms of the second kind without poles except at $\infty$. We fix generators $\alpha_{1}, \alpha_{2}, \beta_{1}, \beta_{2}$ of the fundamental group of $C$ such that their intersections are $\alpha_{i} \cdot \alpha_{j}=\beta_{i} \cdot \beta_{j}=0, \alpha_{i} \cdot \beta_{j}=\delta_{i j}$ for $i, j=1,2$. If we set

$$
\omega^{\prime}=\left[\begin{array}{cc}
\int_{\alpha_{1}} \omega^{(1)} & \int_{\alpha_{2}} \omega^{(1)} \\
\int_{\alpha_{1}} \omega^{(2)} & \int_{\alpha_{2}} \omega^{(2)}
\end{array}\right], \omega^{\prime \prime}=\left[\begin{array}{cc}
\int_{\beta_{1}} \omega^{(1)} & \int_{\beta_{2}} \omega^{(1)} \\
\int_{\beta_{1}} \omega^{(2)} & \int_{\beta_{2}} \omega^{(2)}
\end{array}\right]
$$

the lattice of periods of our Abelian functions appearing below is given by

$$
\Lambda=\omega^{\prime}\left[\begin{array}{l}
\mathbf{Z} \\
\mathbf{Z}
\end{array}\right]+\omega^{\prime \prime}\left[\begin{array}{l}
\mathbf{Z} \\
\mathbf{Z}
\end{array}\right]\left(\subset \mathbf{C}^{2}\right) .
$$

The modulus of $C$ is $Z:=\omega^{\prime-1} \omega^{\prime \prime}$. We also introduce matrices

$$
\eta^{\prime}=\left[\begin{array}{cc}
\int_{\alpha_{1}} \eta^{(1)} & \int_{\alpha_{2}} \eta^{(1)} \\
\int_{\alpha_{1}} \eta^{(2)} & \int_{\alpha_{2}} \eta^{(2)}
\end{array}\right], \eta^{\prime \prime}=\left[\begin{array}{cc}
\int_{\beta_{1}} \eta^{(1)} & \int_{\beta_{2}} \eta^{(1)} \\
\int_{\beta_{1}} \eta^{(2)} & \int_{\beta_{2}} \eta^{(2)}
\end{array}\right] .
$$

Let $J$ be the Jacobian variety of the curve $C$. We identify $J$ with the Picard group $\operatorname{Pic}^{\circ}(C)$ of linear equivalence classes of divisors of degree 0 of $C$. Let $\operatorname{Sym}^{2}(C)$ be the symmetric product of two copies of $C$. Then we have a birational map 


$$
\begin{aligned}
\operatorname{Sym}^{2}(C) & \rightarrow \mathrm{Pic}^{\circ}(C)=J \\
\left(P_{1}, P_{2}\right) & \rightarrow \text { the class of } P_{1}+P_{2}-2 \cdot \infty .
\end{aligned}
$$

We may also identify (the $\mathbf{C}$-rational points of) $J$ with $\mathbf{C}^{2} / \Lambda$. We denote by $\kappa$ the canonical map $\mathbf{C}^{2} \rightarrow \mathbf{C}^{2} / \Lambda$ and by $\iota$ the embedding of $C$ into $J$ given by mapping $P$ to the class of $P-\infty$. Then the image $\iota(C)$ is a theta divisor which is usually denoted by $\Theta$. Although we treat only the case of genus 2, we use both of these two symbols to make as clear as possible the shape of our expected generalization to the case of higher genus. We denote by $O$ the origin of $J$. Obviously $\Lambda=\kappa^{-1}(O)=\kappa^{-1} \iota(\infty)$. If $u \in \mathbf{C}^{2}$, we denote by $u^{(1)}$ and $u^{(2)}$ the first and second entries of $u$.

Lemma 1.1. As a subvariety of $J$, the divisor $\Theta$ is non-singular.

A proof of this fact is seen, for instance, in [19, Lemma 1.7.2, p. 390]. If we set

$$
\delta^{\prime \prime}=\left[\begin{array}{ll}
\frac{1}{2} & \frac{1}{2}
\end{array}\right], \quad \delta^{\prime}=\left[\begin{array}{ll}
0 & \frac{1}{2}
\end{array}\right],
$$

then the sigma function attached to $C$ is defined, as in [2], by

$$
\begin{aligned}
\sigma(u) & =c \exp \left(-\frac{1}{2} u \eta^{\prime} \omega^{\prime-1} t u\right) \\
& \bullet \sum_{n \in \mathbf{Z}^{2}} \exp \left[2 \pi \sqrt{-1}\left\{\frac{1}{2}{ }^{t}\left(n+\delta^{\prime \prime}\right) Z\left(n+\delta^{\prime \prime}\right)+{ }^{t}\left(n+\delta^{\prime \prime}\right)\left(\omega^{\prime-1} t u+\delta^{\prime}\right)\right\}\right] .
\end{aligned}
$$

To fix the constant $c$ above we need the following lemma.

Lemma 1.2. The Taylor expansion of $\sigma(u)$ at $u=(0,0)$ is of the form

$$
u^{(1)}+\frac{1}{6} \lambda_{2} u^{(1)^{3}}-\frac{1}{3} \lambda_{5} u^{(2)^{3}}+\left(d^{\circ}\left(u^{(1)}, u^{(2)}\right) \geq 5\right)
$$

up to a multiplicative constant.

Lemma 1.2 is proved in [4, p. 96] (see also [11, pp. 129-130] or [19, Proposition 2.1.1(2)]). We fix the constant $c$ in (1.1) such that the expansion is exactly of the form in 1.2.

Lemma 1.3. Let $\ell$ be an element in $\Lambda$. The function $u \mapsto \sigma(u)$ on $\mathbf{C}^{2}$ satisfies the translational formula

$$
\sigma(u+\ell)=\chi(\ell) \sigma(u) \exp L(u+\ell, \ell)
$$

where $\chi(\ell)= \pm 1$ is independent of $u, L(u, v)$ is a form which is bilinear over the field of real numbers and $\mathbf{C}$-linear with respect to the first variable $u$ and $L\left(\ell_{1}, \ell_{2}\right)$ is $2 \pi \sqrt{-1}$ times an integer if $\ell_{1}$ and $\ell_{2}$ are in $\Lambda$.

The details of 1.3 are given in [1, p. 286] (see also [19, pp. 395-396]). 
Lemma 1.4. (1) The function $\sigma(u)$ on $C^{2}$ vanishes if and only if $u \in \kappa^{-1}(\Theta)$.

(2) Suppose $v_{1}, v_{2}$ are two points of $\kappa^{-1} \iota(C)$. The function $u \mapsto \sigma\left(u-v_{1}-v_{2}\right)$ for $u \in \kappa^{-1} \iota(C)$ is identically zero if and only if $v_{1}+v_{2}$ is contained in $\kappa^{-1}(O)(=\Lambda)$. If the function is not identically zero, it vanishes only at $u=v_{1}$ and $v_{2}$ modulo $\Lambda$ to order 1 or to order 2 if these two points coincide.

(3) Let $v$ be a fixed point of $\kappa^{-1} \iota(C)$. There exist a point $v_{1}$ of $\kappa^{-1} \iota(C)$ such that the function $u \mapsto \sigma\left(u-v-v_{1}\right)$ on $\kappa^{-1} \iota(C)$ is not identically zero and vanishes at $u=v$ modulo $\Lambda$ of order 1.

The assertions 1.4(1) and (2) are proved in [1, pp. 252-258], for instance. The assertion 1.4(3) obviously follows from (2).

We introduce the functions

$$
\wp_{j k}(u)=-\frac{\partial^{2}}{\partial u^{(j)} \partial u^{(k)}} \log \sigma(u), \wp_{j k \cdots r}(u)=\frac{\partial}{\partial u^{(j)}} \wp_{k \cdots r}(u) .
$$

These functions are periodic with respect to the lattice $\Lambda$ by 1.3 , and have poles along $\kappa^{-1}(\Theta)$ by $1.4(1)$. We also use the notation

$$
\sigma_{j}(u)=\frac{\partial}{\partial u^{(j)}} \sigma(u), \quad \sigma_{j k}(u)=\frac{\partial^{2}}{\partial u^{(j)} \partial u^{(k)}} \sigma(u) .
$$

The following formula was also obtained by Baker in [2, p. 381].

LEMMA 1.5. We have

$$
-\frac{\sigma(u+v) \sigma(u-v)}{\sigma(u)^{2} \sigma(v)^{2}}=\wp_{11}(u)-\wp_{11}(v)+\wp_{12}(u) \wp_{22}(v)-\wp_{12}(v) \wp_{22}(u) .
$$

Let $\left(u^{(1)}, u^{(2)}\right)$ be an arbitrary point not in $\kappa^{-1}(\Theta)$. Then we can find a unique pair of points $\left(x_{1}, y_{1}\right)$ and $\left(x_{2}, y_{2}\right)$ on $C$ such that

$$
u^{(1)}=\int_{\infty}^{\left(x_{1}, y_{1}\right)} \omega^{(1)}+\int_{\infty}^{\left(x_{2}, y_{2}\right)} \omega^{(1)}, \quad u^{(2)}=\int_{\infty}^{\left(x_{1}, y_{1}\right)} \omega^{(2)}+\int_{\infty}^{\left(x_{2}, y_{2}\right)} \omega^{(2)}
$$

with certain choices of the two paths in the integrals. Then one can show that ([2, p. 377])

$$
\wp_{12}(u)=-x_{1} x_{2}, \wp_{22}(u)=x_{1}+x_{2} .
$$

If $u$ is a point on $\kappa^{-1} \iota(C)$, the $x$ - and $y$-coordinates of $\iota^{-1} \kappa(u)$ will be denoted by $x(u)$ and $y(u)$, respectively.

LEMMA 1.6. If $u$ is a point on $\kappa^{-1} \iota(C)$, then

$$
\frac{\wp_{12}}{\wp_{22}}(u)=\frac{\sigma_{1}}{\sigma_{2}}(u)=-x(u)
$$

These equalities are shown in [12, p. 124] by using 1.4(1) and (1.3) (see also [19, proof of Proposition 2.1.1]). By 1.2 and 1.4(1), we see the following. 
LEMMA 1.7. If $u \in \kappa^{-1} \iota(C)$, then

$$
u^{(1)}=\frac{1}{3} u^{(2)^{3}}+\left(d^{\circ}\left(u^{(2)}\right) \geq 4\right) .
$$

Hence $u^{(2)}$ is a local parameter at $(0,0)$. While Lemma 1.8 below gives a local parameter at any point of $\kappa^{-1} \iota(C)$ except $\kappa^{-1}(O)$ and is not used in this paper, it would be helpful to the reader's understanding of our main results.

Lemma 1.8. Let $v_{0}=\left(v_{0}^{(1)}, v_{0}^{(2)}\right)$ be a fixed point on $\kappa^{-1} \iota(C)$ and assume $v_{0} \notin \kappa^{-1}(O)$. Let $u=\left(u^{(1)}, u^{(2)}\right)$ be a variable point on $\kappa^{-1} \iota(C)$. Then the variable $u^{(1)}-v_{0}^{(1)}$ is a local parameter at $v_{0}$ along $\kappa^{-1} \iota(C)$. In other words, the function $u \mapsto u^{(1)}-v_{0}^{(1)}$ vanishes at $u=v_{0}$ of order 1 .

Proof. Since

$$
\frac{d\left(u^{(2)}-v_{0}^{(2)}\right)}{d\left(u^{(1)}-v_{0}^{(1)}\right)}=\frac{d u^{(2)}}{d u^{(1)}}=\frac{d u^{(2)}}{d x} \frac{d x}{d u^{(1)}}=x(u),
$$

we have

$$
u^{(2)}-v_{0}^{(2)}=x\left(v_{0}\right)\left(u^{(1)}-v_{0}^{(1)}\right)+\left(d^{\circ}\left(u^{(1)}-v_{0}^{(1)}\right) \geq 2\right) .
$$

Lemma 1.4(3) states that there exists a point $v_{1}$ in $\kappa^{-1} \iota(C)$ such that the function $u \mapsto \sigma\left(u-v_{0}-v_{1}\right)$ is not identically zero and vanishes at $u=v_{0}$ of order 1 . Then we have

$$
\begin{aligned}
\sigma\left(u-v_{0}-v_{1}\right)= & \sigma_{1}\left(-v_{1}\right)\left(u^{(1)}-v_{0}^{(1)}\right)+\sigma_{2}\left(-v_{1}\right)\left(u^{(2)}-v_{0}^{(2)}\right) \\
& +\left(d^{\circ}\left(u^{(1)}-v_{0}^{(1)}, u^{(2)}-v_{0}^{(2)}\right) \geq 2\right) .
\end{aligned}
$$

Hence the vanishing order of $u \mapsto u^{(1)}-v_{0}^{(1)}$ at $u=v_{0}$ cannot be higher than 1 . So we see that $u^{(1)}-v_{0}^{(1)}$ is a local parameter at $v_{0}$.

Lemma 1.9. (1) Let $u$ be an arbitrary point on $\kappa^{-1} \iota(C)$. Then $\sigma_{2}(u)$ is 0 if and only if $u$ is a lattice point, that is, the case $\kappa(u)=\iota(\infty)$.

(2) The Taylor expansion of the function $\sigma_{2}(u)$ on $\kappa^{-1} \iota(C)$ at $u=(0,0)$ is of the form

$$
\sigma_{2}(u)=-u^{(2)^{2}}+\left(d^{\circ}\left(u^{(2)}\right) \geq 3\right) .
$$

(3) Let $v_{0}=\left(v_{0}^{(1)}, v_{0}^{(2)}\right)$ be a fixed point on $\kappa^{-1} \iota(C)$ and assume $v_{0} \notin \kappa^{-1}(O)$. The Taylor expansion of the function $u \rightarrow \sigma\left(u-v_{0}\right)$ on $\kappa^{-1} \iota(C)$ at $u=(0,0)$ is of the form

$$
\sigma\left(u-v_{0}\right)=\sigma_{2}\left(v_{0}\right) u^{(2)}+\left(d^{\circ}\left(u^{(2)}\right) \geq 2\right) .
$$

Proof. If $\sigma_{2}(u)=0$, then the second equality of 1.6 yields $\sigma_{1}(u)=0$. This contradicts 1.4(1), (2) and 1.1. So $\sigma_{2}(u) \neq 0$. The assertion (2) follows from 1.2 and 1.7. Because $\sigma_{2}(u)$ is an even function, the assertion (3) follows from 1.4(1) and 1.7. 
Proposition 1.10. Let $n$ be a positive integer. If $u \in \kappa^{-1} \iota(C)$, then

$$
\psi_{n}(u):=\frac{\sigma(n u)}{\sigma_{2}(u)^{n^{2}}}
$$

is periodic with respect to $\Lambda$. In other words it is a function on $\iota(C)$.

For a proof of this, see [12, p. 124] or [19, Proposition 3.2.2].

LEMMA 1.11. We have that $\psi_{2}(u)=2 y(u)$.

This is a result stated in [12, p. 128] (see also [19, Lemma 3.2.4]). We end this Section by stating the following easily shown relations (see [19, Lemma 2.3.1]).

LEMMA 1.12. If $u \in \kappa^{-1} \iota(C)$ then

$$
x(u)=\frac{1}{u^{(2)^{2}}}+\left(d^{\circ}\left(u^{(2)}\right) \geq-1\right), \quad y(u)=-\frac{1}{u^{(2)^{5}}}+\left(d^{\circ}\left(u^{(2)}\right) \geq-4\right) .
$$

2. A generalization of the formula of Frobenius-Stickelberger. Let us start by stating the simplest case of our generalization.

Proposition 2.1. Assume $u$ and $v$ belong to $\kappa^{-1} \iota(C)$. Then

$$
-\frac{\sigma(u+v) \sigma(u-v)}{\sigma_{2}(u)^{2} \sigma_{2}(v)^{2}}=-x(u)+x(v)\left(=\left|\begin{array}{cc}
1 & x(u) \\
1 & x(v)
\end{array}\right|\right) .
$$

Proof. We give two proofs. First we use the formula of 1.5. For $u \notin \kappa^{-1} \iota(C)$ and $v \notin \kappa^{-1} \iota(C)$, after dividing the formula of 1.5 by

$$
\wp_{22}(u) \wp_{22}(v)=\frac{\left(\sigma_{2}(u)^{2}-\sigma_{22}(u) \sigma(u)\right)}{\sigma(u)^{2}} \cdot \frac{\left(\sigma_{2}(v)^{2}-\sigma_{22}(v) \sigma(v)\right)}{\sigma(v)^{2}},
$$

and bringing $u$ and $v$ close to any points on $\kappa^{-1} \iota(C)$, we have the desired formula because of 1.4(1) and 1.6.

Our second proof is done by comparing the zeros and poles of each side. If we regard the two sides as functions of $u$, they are functions on $\iota(C)$. We may assume $v \notin \kappa^{-1}(O)$. The zeros of the two sides coincide and they are at $u=v$ and at $u=-v$, and both sides have their only pole at $u=(0,0)$ of order 2 by $1.9(2)$ and 1.12 . By $1.9(3)$, the coefficient of $1 / u^{(2)^{2}}$ of the left hand side is -1 . Thus we have proved the formula once again.

REMARK 2.2. Although the formula of 1.5 is a natural generalization of the corresponding formula for Weierstrass' functions $\sigma(u)$ and $\wp(u)$ ([21, p. 451, Example 1]), the formula of 2.1 bears a striking likeness to the formula for elliptic functions. Finding this formula was the clue to the discovery of the formula in Theorem 2.3. 
Our generalization of $(0.2)$ is the following formula.

Theorem 2.3. Let $n$ be a positive integer. Assume $u\left(=u_{0}\right), u_{1}, \cdots, u_{n}$ all belong to $\kappa^{-1} \iota(C)$. Then

$$
-\frac{\sigma\left(u_{0}+u_{1}+\cdots+u_{n}\right) \prod_{i<j} \sigma\left(u_{i}-u_{j}\right)}{\sigma_{2}\left(u_{0}\right)^{n+1} \sigma_{2}\left(u_{1}\right)^{n+1} \cdots \sigma_{2}\left(u_{n}\right)^{n+1}}
$$

is equal to

$$
\left|\begin{array}{ccccccccc}
1 & x\left(u_{0}\right) & x^{2}\left(u_{0}\right) & y\left(u_{0}\right) & x^{3}\left(u_{0}\right) & y x\left(u_{0}\right) & \cdots & y x^{(n-4) / 2}\left(u_{0}\right) & x^{(n+2) / 2}\left(u_{0}\right) \\
1 & x\left(u_{1}\right) & x^{2}\left(u_{1}\right) & y\left(u_{1}\right) & x^{3}\left(u_{1}\right) & y x\left(u_{1}\right) & \cdots & y x^{(n-4) / 2}\left(u_{1}\right) & x^{(n+2) / 2}\left(u_{1}\right) \\
\vdots & \vdots & \vdots & \vdots & \vdots & \vdots & \ddots & \vdots & \vdots \\
1 & x\left(u_{n}\right) & x^{2}\left(u_{n}\right) & y\left(u_{n}\right) & x^{3}\left(u_{n}\right) & y x\left(u_{n}\right) & \cdots & y x^{(n-4) / 2}\left(u_{n}\right) & x^{(n+2) / 2}\left(u_{n}\right)
\end{array}\right|
$$

or

$$
\left|\begin{array}{ccccccccc}
1 & x\left(u_{0}\right) & x^{2}\left(u_{0}\right) & y\left(u_{0}\right) & x^{3}\left(u_{0}\right) & y x\left(u_{0}\right) & \cdots & x^{(n+1) / 2}\left(u_{0}\right) & y x^{(n-3) / 2}\left(u_{0}\right) \\
1 & x\left(u_{1}\right) & x^{2}\left(u_{1}\right) & y\left(u_{1}\right) & x^{3}\left(u_{1}\right) & y x\left(u_{1}\right) & \cdots & x^{(n+1) / 2}\left(u_{1}\right) & y x^{(n-3) / 2}\left(u_{1}\right) \\
\vdots & \vdots & \vdots & \vdots & \vdots & \vdots & \ddots & \vdots & \vdots \\
1 & x\left(u_{n}\right) & x^{2}\left(u_{n}\right) & y\left(u_{n}\right) & x^{3}\left(u_{n}\right) & y x\left(u_{n}\right) & \cdots & x^{(n+1) / 2}\left(u_{n}\right) & y x^{(n-3) / 2}\left(u_{n}\right)
\end{array}\right|
$$

according to whether $n$ is even or odd.

Proof. Proposition 2.1 is the case of $n=1$. We prove this formula by induction on $n$. Suppose the points $u_{0}, u_{1}, \cdots, u_{g}$ do not belong to $\kappa^{-1} \iota(C)$. We know that each of the functions

$$
u_{j} \mapsto-\frac{\sigma\left(u_{0}+u_{1}+\cdots+u_{n}\right) \prod_{i<j} \sigma\left(u_{i}-u_{j}\right)}{\sigma\left(u_{0}\right)^{n+1} \sigma\left(u_{1}\right)^{n+1} \cdots \sigma\left(u_{n}\right)^{n+1}}
$$

is periodic with respect to $\Lambda$ by $1.4(1)$ and the theorem of the square ([18, Corollory 4 on p. 59]). After multiplying

$$
\begin{aligned}
& (-1)^{n+1} \frac{\wp_{22}\left(u_{0}\right) \wp_{22}\left(u_{1}\right) \cdots \wp_{22}\left(u_{n}\right)}{\wp_{222}\left(u_{0}\right) \wp_{222}\left(u_{1}\right) \cdots \wp_{222}\left(u_{n}\right)} \\
& =\frac{\left(\sigma_{2}^{2}-\sigma_{22}\right) \sigma}{\sigma_{2}^{3}-3 \sigma \sigma_{2} \sigma_{22}+\sigma \sigma_{222}}\left(u_{0}\right) \frac{\left(\sigma_{2}^{2}-\sigma_{22}\right) \sigma}{\sigma_{2}^{3}-3 \sigma \sigma_{2} \sigma_{22}+\sigma \sigma_{222}}\left(u_{1}\right) \cdots \frac{\left(\sigma_{2}^{2}-\sigma_{22}\right) \sigma}{\sigma_{2}^{3}-3 \sigma \sigma_{2} \sigma_{22}+\sigma \sigma_{222}}\left(u_{n}\right),
\end{aligned}
$$

bringing $u_{j}$ 's close to any point of $\kappa^{-1} \iota(C)$, we see by $1.4(1)$ that the left hand side of the claimed formula is, as a function of each $u_{j}$, a periodic function on $\kappa^{-1} \iota(C)$. Now we regard both sides of the claimed formula as a function on $C$ of variable $u=u_{0}$, and regard the points $u_{j}$ as points on $C$. We denote the left hand side by $f_{1}(u)$ and the right hand side by $f_{2}(u)$. We consider a divisor

$$
D=(n+2) \infty-u_{1}-u_{2}-\cdots-u_{n}
$$

of $C$. Lemmas $1.4(2)$ and 1.9 show that the divisor $\left(f_{1}\right)+D$ is effective, and 1.12 shows that the divisor $\left(f_{2}\right)+D$ is also effective. Since $D$ is not a canonical divisor and 
$C$ is of genus 2, the Riemann-Roch theorem shows that the space of the functions $f$ such that $(f)+D$ is effective is of dimension 1 . Therefore the two sides coincide up to a non-zero multiplicative constant. We know by 1.4(2), 1.9(2) and 1.9(3) that the coefficient of the Laurent expansion at $u=(0,0)$ with respect to $u^{(g)}$ of the left hand side is just the left hand side of the hypothetical statement of our induction. We also know by 1.12 that the coefficient of such the Laurent expansion of the right hand side is just the right hand side of the hypothesis. Thus the formula holds.

REMARK 2.4. The formula in 2.3 should be regarded as a generalization of the genus-one formula

$$
\begin{aligned}
& \frac{\sigma\left(u_{0}+u_{1}+\cdots+u_{n}\right) \prod_{i<j} \sigma\left(u_{i}-u_{j}\right)}{\sigma\left(u_{0}\right)^{n+1} \sigma\left(u_{1}\right)^{n+1} \cdots \sigma\left(u_{n}\right)^{n+1}} \\
& =\left|\begin{array}{ccccccc}
1 & x\left(u_{0}\right) & y\left(u_{0}\right) & x^{2}\left(u_{0}\right) & y x\left(u_{0}\right) & x^{3}\left(u_{0}\right) & \cdots \\
1 & x\left(u_{1}\right) & y\left(u_{1}\right) & x^{2}\left(u_{2}\right) & y x\left(u_{2}\right) & x^{3}\left(u_{1}\right) & \cdots \\
\vdots & \vdots & \vdots & \vdots & \vdots & \vdots & \ddots \\
1 & x\left(u_{n}\right) & y\left(u_{n}\right) & x^{2}\left(u_{n}\right) & y x\left(u_{n}\right) & x^{3}\left(u_{n}\right) & \cdots
\end{array}\right|
\end{aligned}
$$

rather than of $(0.1)$. Here we suppose the sigma function $\sigma(u)$ is associated with the elliptic curve defined by an equation of the form $y^{2}=x^{3}+\lambda_{2} x^{2}+\lambda_{1} x+\lambda_{0}$. The size of the matrix above is also $n+1$ by $n+1$. This formula is easily obtained from $(0.1)$ and explains the meaning of the multiplicative constant of the left hand side of (0.1).

3. A determinant expression for generalized psi-functions. We give in this Section a generalization of the formula of Kiepert [14] (see also [21, Exercise 33, p. 460] or $[9$, p. 186]). There is a pretty formula:

Lemma 3.1. Let $u$ and $v$ be belong to $\kappa^{-1} \iota(C)$. Then

$$
\lim _{u^{(1)} \rightarrow v^{(1)}} \frac{\sigma(u-v)}{u^{(1)}-v^{(1)}}=1 .
$$

Proof. Because of 2.1 we have

$$
\frac{x(u)-x(v)}{u^{(1)}-v^{(1)}}=\frac{\sigma(u+v)}{\sigma_{2}(u)^{2} \sigma_{2}(v)^{2}} \cdot \frac{\sigma(u-v)}{u^{(1)}-v^{(1)}} .
$$

Now we bring $u^{(1)}$ close to $v^{(1)}$. Then the limit of the left hand side is

$$
\lim _{u^{(1)} \rightarrow v^{(1)}} \frac{x(u)-x(v)}{u^{(1)}-v^{(1)}}=\frac{d x}{d u^{(1)}}(v) .
$$

This is equal to $2 y$ by (1.2). The required formula follows from 1.11.

REMARK 3.2. The reader should take care to note that the left hand side in 3.1 is not 


$$
\lim _{h^{(1)} \rightarrow 0} \frac{\sigma(h)}{h^{(1)}}\left(h \in \kappa^{-1} \iota(C)\right) .
$$

Our generalization of the formula $(0.2)$ for $\psi_{n}(u)\left(=\sigma(n u) / \sigma_{2}(u)^{n^{2}}\right)$ is the following.

Theorem 3.3. Let $n$ be an integer greater than 1. Assume that $u$ belongs to $\kappa^{-1} \iota(C)$. Let $j$ be 1 or 2 . Then the following formula holds:

$-(1 ! 2 ! \cdots(n-1) !) \psi_{n}(u)=x^{(j-1) n(n-1) / 2}(u) \times$

$\begin{array}{|ccccccc|}x^{\prime}(u) & \left(x^{2}\right)^{\prime}(u) & y^{\prime}(u) & \left(x^{3}\right)^{\prime}(u) & (y x)^{\prime}(u) & \left(x^{4}\right)^{\prime}(u) & \ldots \\ x^{\prime \prime}(u) & \left(x^{2}\right)^{\prime \prime}(u) & y^{\prime \prime}(u) & \left(x^{3}\right)^{\prime \prime}(u) & (y x)^{\prime \prime}(u) & \left(x^{4}\right)^{\prime \prime}(u) & \ldots \\ x^{\prime \prime \prime}(u) & \left(x^{2}\right)^{\prime \prime \prime}(u) & y^{\prime \prime \prime}(u) & \left(x^{3}\right)^{\prime \prime \prime}(u) & (y x)^{\prime \prime \prime}(u) & \left(x^{4}\right)^{\prime \prime \prime}(u) & \ldots \\ \vdots & \vdots & \vdots & \vdots & \vdots & \vdots & \ddots \\ x^{(n-1)}(u) & \left(x^{2}\right)^{(n-1)}(u) & y^{(n-1)}(u) & \left(x^{3}\right)^{(n-1)}(u) & (y x)^{(n-1)}(u) & \left(x^{4}\right)^{(n-1)}(u) & \ldots\end{array} \mid$

Here the size of the matrix is $n-1$ by $n-1$. The symbols ', " $, \ldots,{ }^{(n-1)}$ denote $\frac{d}{d u^{(j)}}$, $\left(\frac{d}{d u^{(j)}}\right)^{2}, \cdots,\left(\frac{d}{d u^{(j)}}\right)^{n-1}$, respectively.

Proof. We start with replacing $n$ by $n-1$ in 2.3. If we use $\frac{d}{d u^{(1)}}=x(u) \frac{d}{d u^{(2)}}$ in the following proof for the case of $j=1$, we can easily check that the case of $j=2$ also holds. We may assume that $u \neq(0,0)$ modulo $\Lambda$. For a fixed $u$, we write $h=\left(h^{(1)}, h^{(2)}\right)=u_{1}-u$ and varies $u_{1}$. Then $h^{(1)}$ is a local parameter at the point $u$ by 1.9 and the right hand side of 2.3 is equal to

$$
\begin{aligned}
& \left|\begin{array}{cccc}
1 & x(u) & x^{2}(u) & \cdots \\
0 & x(u+h)-x(u) & x^{2}(u+h)-x^{2}(u) & \cdots \\
1 & x\left(u_{2}\right) & x^{2}\left(u_{2}\right) & \cdots \\
\vdots & \vdots & \vdots & \ddots
\end{array}\right| \\
& =\left|\begin{array}{cccc}
1 & x(u) & x^{2}(u) & \\
0 & x^{\prime}(u) h^{(1)}+\left(d^{\circ}\left(h^{(1)}\right) \geq 2\right) & \left(x^{2}\right)^{\prime}(u) h^{(1)}+\left(d^{\circ}\left(h^{(1)}\right) \geq 2\right) & \cdots \\
1 & x\left(u_{2}\right) & x^{2}\left(u_{2}\right) & \cdots \\
\vdots & \vdots & \vdots & \ddots
\end{array}\right|
\end{aligned}
$$

by Taylor's theorem in one variable. After dividing both sides by $h^{(1)}$ and taking the limit when $h^{(1)} \rightarrow 0$ while keeping $u+h \in \kappa^{-1} \iota(C)$, by using 3.1 , we arrive at the formula

$$
\begin{aligned}
& -\frac{\sigma\left(2 u+u_{2}+\cdots+u_{n-1}\right) \sigma\left(u-u_{2}\right)^{2} \cdots \sigma\left(u-u_{n-1}\right)^{2} \prod_{2 \leq i<j} \sigma\left(u_{i}-u_{j}\right)}{\sigma_{2}(u)^{2 n} \sigma_{2}\left(u_{2}\right)^{n} \cdots \sigma_{2}\left(u_{n-1}\right)^{n}} \\
& =\left|\begin{array}{cccc}
1 & x(u) & x^{2}(u) & \cdots \\
0 & x^{\prime}(u) & \left(x^{2}\right)^{\prime}(u) & \cdots \\
1 & x\left(u_{2}\right) & x^{2}\left(u_{2}\right) & \cdots \\
\vdots & \vdots & \vdots & \ddots
\end{array}\right| .
\end{aligned}
$$


Applying Taylor's theorem in one variable again we see that the right hand side with $h=u_{2}-u$ is given by

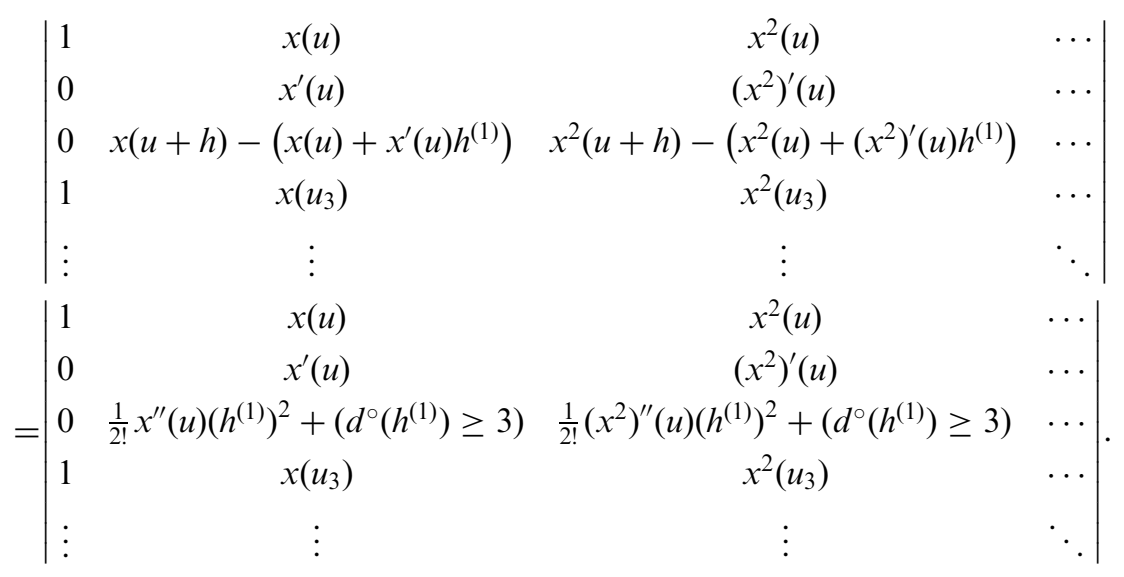

After dividing both sides by $\left(h^{(1)}\right)^{2}$ and taking the limit when $h^{(1)} \rightarrow 0$, by applying 3.1 we have

$$
\begin{aligned}
& -(1 ! 2 !) \frac{\sigma\left(3 u+u_{3}+\cdots+u_{n-1}\right) \sigma\left(u-u_{3}\right)^{3} \cdots \sigma\left(u-u_{n-1}\right)^{3} \prod_{3 \leq i<j} \sigma\left(u_{i}-u_{j}\right)}{\sigma_{2}(u)^{3 n} \sigma_{2}\left(u_{3}\right)^{n} \cdots \sigma_{2}\left(u_{n-1}\right)^{n}} \\
& =\left|\begin{array}{cccc}
1 & x(u) & x^{2}(u) & \cdots \\
0 & x^{\prime}(u) & \left(x^{2}\right)^{\prime}(u) & \cdots \\
0 & x^{\prime \prime}(u) & \left(x^{2}\right)^{\prime \prime}(u) & \cdots \\
1 & x\left(u_{3}\right) & x^{2}\left(u_{3}\right) & \cdots \\
\vdots & \vdots & \vdots & \ddots
\end{array}\right|
\end{aligned}
$$

Using similar operations repeatedly, we have the formula for $\psi_{n}(u)$.

EXAMPLE. We have

$$
\begin{aligned}
x^{\prime}(u) & =\frac{d}{d u^{(1)}} x(u)=1 / \frac{d u^{(1)}}{d x}(u)=\frac{2 y}{x}(u), \quad y^{\prime}(u)=\frac{D f(x)}{x}, \\
x^{\prime \prime}(u) & =\frac{d}{d u^{(1)}}\left(\frac{2 y}{x}\right)=2 \frac{x \cdot D f(x)-2 y^{2}}{x^{3}}, \quad\left(x^{2}\right)^{\prime}(u)=4 y(u), \quad\left(x^{2}\right)^{\prime \prime}(u)=\frac{4}{x} D f(x),
\end{aligned}
$$

where $f(x)$ is as in the beginning of Section 1 and $D f(x)=\frac{d}{d x} f(x)$. Then $-\psi_{3}(u)=-\sigma(3 u) / \sigma_{2}(u)^{9}=8 y^{3}(u)$ by 3.3. This example is mentioned in the proof of [12, Lemma 2 (e)], too, and the proof here is different from the one there (see also [19, Lemma 3.2(2)]). 


\section{REFERENCES}

1. H. F. Baker, Abelian functions - Abel's theorem and the allied theory including the theory of the theta functions (Cambridge University Press, 1897; reprint, 1995). 384 .

2. H. F. Baker, On the hyperelliptic sigma functions, Amer. J. Math. 20 (1898), 301-

3. H. F. Baker, On a system of differential equations leading to periodic functions, Acta Math. 27 (1903), 135-156.

4. H. F. Baker, An introduction to the theory of multiply periodic functions (Cambridge University Press, 1907).

5. V. M. Buchstaber, V. Z. Enolskii and D. V. Leykin, Kleinian functions, hyperelliptic Jacobians and applications, Reviews in Math. and Math. Physics 10 (1997), 1-125.

6. F. Brioschi, Sur quelques formules pour la multiplication des fonctions elliptiques, $C$. R. Acad. Sci. Paris 59 (1864), 769-775.

7. D. G. Cantor, On the analogue of the division polynomials for hyperelliptic curves, $J$. Reine Angew. Math. 447 (1994), 91-145.

8. J. Fay, Theta functions on Riemann surfaces, Lecture Notes in Math. No. 352, (Springer-Verlag, 1973). 1922)

9. R. Fricke, Die elliptishen Functionen und ihre Anwendungen, I, II (Teubner, 1916,

10. F. G. Frobenius and L. Stickelberger, Zur Theorie der elliptischen Functionen, $J$. Reine Angew. Math. 83 (1877), 175-179.

11. D. Grant, On a generalization of Jacobi's derivative formula to dimension two, $J$. Reine Angew. Math. 392 (1988), 125-136.

12. D. Grant, A generalization of a formula of Eisenstein, Proc. London Math. Soc. 62 (1991), 121-132.

13. D. Grant, On Gunning's prime form in genus 2, Canadian Math. Bull. (to appear).

14. L. Kiepert, Wirkliche Ausführung der ganzzahligen Multiplikation der elliptichen Funktionen, J. Reine Angew. Math. 76 (1873), 21-33.

15. S. Matsutani and Y. Onishi, Determinant expression for hyperelliptic functions, Preprint, http://arxiv.org/abs/math.NT/0105189 (2001).

16. C. R. Matthews, Gauss sums and elliptic functions : I. The Kummer Sum, Invent. Math. 52 (1979), 163-185.

17. C. R. Matthews, Gauss sums and elliptic functions : II. The Quartic Sum, Invent. Math. 54 (1979), 23-52.

18. D. Mumford, Abelian varieties (Oxford University Press, 1985).

19. Y. Ônishi, Complex multiplication formulae for hyperelliptic curves of genus three, Tokyo J. Math. 21 (1998), 381-431.

20. H. Weber, Lehrbuch der Algebra III (F. Vieweg, 1908; Chelsea, 1961).

21. E. T. Whittaker and G. N. Watson, A course of modern analysis (Cambridge University Press, 1902). 